room for hospitals to undertake practices that will markedly decrease their VAP rates and yet do little or nothing to improve patient outcomes. ${ }^{12}$

The implications are as follows: (1) VAP as currently defined is not a suitable measure for benchmarking hospitals or for external assessment of quality of care since the diagnosis is too inexact and liable to be gamed; (2) internal quality improvement initiatives to decrease VAP rates need to pay close attention to the diagnostic protocol to ensure consistency over time; and (3) we need a new, more objective and accurate measure for quality of care in ventilated patients that will reliably predict patients' outcomes. Until then, quality improvement initiatives would do well to track directly patient outcomes, such as duration of ventilation, length of stay and mortality, to increase confidence that an observed change in VAP rates translates into improved patient outcomes. This is critical to avoid unintended consequences from well-meaning initiatives to drive our hospital-acquired infection rates to zero.

Acknowledgements: The author wishes to thank Richard Platt MD, MSc for thoughtful comments on an earlier version of this manuscript.

Competing interests: None.

Thorax 2009;64:463-465. doi:10.1136/thx.2009.114165

\section{REFERENCES}

1. Conway Morris A, Kefala K, Simpson AJ, et al. Evaluation of the effect diagnostic methodology on the reported incidence of ventilator-associated pneumonia. Thorax 2009;64:516-22.

2. Kirtland $\mathbf{S H}$, Corley DE, Winterbauer $\mathrm{RH}$, et al. The diagnosis of ventilator-associated pneumonia: a comparison of histologic, microbiologic, and clinical criteria. Chest 1997;112:445-57.

3. Papazian L, Thomas P, Garbe L, et al. Bronchoscopic or blind sampling techniques for the diagnosis of ventilator-associated pneumonia. Am J Respir Crit Care Med 1995;152:1982-91

4. Fabregas N, Ewig S, Torres A, et al. Clinical diagnosis of ventilator associated pneumonia revisited: comparative validation using immediate post-mortem lung biopsies. Thorax 1999;54:867-73.

5. Marquette $\mathbf{C H}$, Copin MC, Wallet F, et al. Diagnostic tests for pneumonia in ventilated patients: prospective evaluation of diagnostic accuracy using histology as a diagnostic gold standard. Am J Respir Crit Care Med 1995;151:1878-88

6. Torres A, el-Ebiary M, Padro L, et al. Validation of different techniques for the diagnosis of ventilatorassociated pneumonia. Comparison with immediate postmortem pulmonary biopsy. Am J Respir Crit Care Med 1994;149:324-31.

7. Klompas M, Kulldorff M, Platt R. Risk of misleading ventilator-associated pneumonia rates with use of standard clinical and microbiological criteria. Clin Infect Dis 2008;46:1443-6.

8. Schurink CA, Van Nieuwenhoven CA, Jacobs JA, et al. Clinical pulmonary infection score for ventilator-associated pneumonia: accuracy and inter-observer variability. Intensive Care Med 2004; 30:217-24.

9. Fagon JY, Chastre J, Wolff M, et al. Invasive and noninvasive strategies for management of suspected ventilator-associated pneumonia. A randomized trial. Ann Intern Med 2000;132:621-30.

10. Shorr AF, Sherner JH, Jackson WL, et al. Invasive approaches to the diagnosis of ventilator-associated pneumonia: a meta-analysis. Crit Care Med 2005; 33:46-53.

11. Heyland D, Dodek P, Muscedere J, et al. A randomized trial of diagnostic techniques for ventilator-associated pneumonia. N Engl J Med 2006;355:2619-30.

12. Klompas M, Platt R. Ventilator-associated pneumonia - the wrong quality measure for benchmarking. Ann Intern Med 2007;147:803-5.

\title{
The fruits of our efforts: time for a different view of lung cancer and CT screening
}

\section{Frank Detterbeck}

Lung cancer continues to be by far the leading cause of cancer deaths, primarily because it is usually not found until it is in a relatively advanced stage. As a result, a great deal of effort has focused on using CT imaging to screen a broad population. The two most influential papers on CT screening for lung cancer published recently have drawn conclusions that are diametrically opposed. ${ }^{12}$ Henschke et al ${ }^{1}$ found compelling evidence that CT screening would save many patients from death, while Bach et al concluded that CT screening may lead to harm through overtreatment of inconsequential lung cancers. It is worth taking a closer look at how two thoughtful groups can arrive at such disparate views.

The paper by Henschke et a $l^{1}$ reported a 5 -year survival rate of $86 \%$ for patients with lung cancer detected by CT screening. This is dramatically better than the

Correspondence to: Dr F C Detterbeck, Yale University, New Haven, MA 06520-8062, USA; frank.detterbeck@ yale.edu
5 -year survival rate of $16 \%$ for patients with lung cancer detected by routine care and reported through the US national cancer database. ${ }^{3}$ The implication is that CT screening has changed the outcome of the patients by early detection and early initiation of treatment.

The paper by Bach et al compared the results of three single-arm CT screening studies with predicted results using a model derived from and validated in patients with lung cancer detected during routine care as it exists currently. The number of lung cancers and lung cancer resections that actually occurred in the CT screened cohort was much higher than what was predicted by the model, whereas the number of deaths from lung cancer matched closely. The authors therefore concluded that CT screening resulted in overdiagnosis and overtreatment of indolent lung cancers that are of no clinical consequence in a substantial proportion of patients without any benefit. ${ }^{2}$

Some of the discussion of these papers centred around funding sources and whether there was potential for a bias as a result. ${ }^{4}$ The real issue, however, is to understand why the data from these two studies seem so dissimilar.

An emerging concept is that lung cancer involves a spectrum of tumours ranging from some that are very aggressive to others that are very indolent..$^{5-10}$ Importantly, it is becoming clear that CT screening in particular selects a population of patients that has a different spectrum of disease than that of patients detected by routine care. For example, a systematic review showed that the average volume doubling time of lung cancers detected during routine care was approximately 135 days compared with almost 500 days for lung cancers detected by CT screening. ${ }^{5}$ The proportion of patients with long doubling times (>400 days) increased from $3 \%$ in patients detected by routine care to $27 \%$ in those detected by CT screening. ${ }^{5-10}$ Thus, a comparison of lung cancers detected by CT screening with those detected by routine care is like comparing apples with oranges.

The comparison inherent in the paper by Henschke et $a l^{1}$ between lung cancers detected by CT screening and those detected during routine care is therefore flawed if the spectrum of disease is different in these two cohorts. Metaphorically, it is inappropriate to conclude from the observed differences that CT screening converted apples into oranges. Instead, one should conclude 
that the process of CT screening identified many oranges instead of the apples we were accustomed to.

Bach et $a l^{2}$ recognise that cohorts detected by CT screening are different from those detected by routine care, but applied the same management to both groups. This is somewhat like recognising that oranges are different from apples but using a recipe for apple pie for both. Using this approach leads to the observation that apple pie made with oranges is not desirable.

The underlying problem in the study by Henschke et al lies in viewing lung cancer in a black and white manner; any lung cancer is rapidly fatal. Henschke et al do report that the eight patients diagnosed with lung cancer via CT screening who were not treated died, although no details about these patients are provided (such as cause of death). ${ }^{1}$ This is consistent with the natural history of lung cancer detected by routine care (5-year survival of $2 \%$ for untreated patients with stage I lung cancer). ${ }^{5}$ However, the black and white view of lung cancer is countered by the consistent finding that there is a spectrum of doubling times among lung cancers, and that this spectrum is markedly shifted to a higher proportion of patients with very long doubling times in tumours detected by CT screening compared with those detected by routine care. ${ }^{5}$

The paper by Bach et al suffers from a similar black and white manner of thinking: a lung cancer is either a serious disease (requiring the "standard" recipe of treatment) or it is overdiagnosed (and overtreated if the standard approach is used). Much attention has been focused on overdiagnosis bias, meaning patients who are diagnosed with an indolent tumour that has no effect on their length of life (ie, they will die of other causes and not the cancer). Much less attention has been paid to length bias, which occurs when the process of screening selects a higher proportion of patients with less aggressive cancers. A factor contributing to this may be that the term "length bias" is less intuitive and less well understood than lead time bias or overdiagnosis bias. A more intuitive term for length bias may be "spectrum bias". Nevertheless, death and therefore also mortality of patients with lung cancer remains an unassailable end point, making overdiagnosis an issue that cannot be easily brushed aside.

As in most conflicts, it may be that, in some ways, Henschke et al and Bach et al are both right and in some ways they are both wrong. The difficulty in defining the role of CT screening may arise because we are not formulating the questions appropriately. Perhaps it is time to view lung cancer in a more nuanced fashion as a disease that has a wide spectrum of behaviour. If the spectrum of disease is varied, perhaps the treatment should also be varied (ie, sublobar resection, radiofrequency ablation, stereotactic radiosurgery or simply careful observation with subsequent intervention if needed). Perhaps the outcome measures also need to be reassessed (eg, 5-year survival may not be an appropriate measure for indolent tumours).

At present our ability to predict the behaviour of lung cancers is limited, and therefore so is our ability to clearly define new treatment approaches and outcome measures. Nevertheless, it appears that the time has come to address these questions, paying careful attention to the characteristics of the patients involved. The spectrum of disease may be changing not only in CT screening programmes but also in the general population due to an increased prevalence of CT scanning in general. ${ }^{11}$ A binary black and white view of the biological behaviour and the approach to treatment of lung cancer may be inhibiting us from a full understanding of how best to approach patients and how a high prevalence of CT scanning affects this.

Competing interests: None.

Thorax 2009;64:465-466. doi:10.1136/thx.2008.106328

\section{REFERENCES}

1. Henschke C, Yankelevitz D, Libby DM, et al. Survival of patients with stage I lung cancer detected on CT screening. N Engl J Med 2006;355:1763-71.

2. Bach PB, Jett JR, Pastorino U, et al. Computed tomography screening and lung cancer outcomes. JAMA 2007;297:953-61.

3. Jemal A, Siegel R, Ward E, et al. Cancer statistics, 2007. CA 2007;57:43-66.

4. Henschke CI, Yankelevitz DF. Unreported financial disclosures. JAMA 2008;299:1770.

5. Detterbeck F, Gibson C. Turning gray: the natural history of lung cancer over time. J Thorac Oncol 2008;3:781-92

6. Kerr K, Lamb D. Actual growth rate and tumour cell proliferation in human pulmonary neoplasms. Br J Cancer 1984;50:343-9.

7. Arai T, Kuroishi T, Saito Y, et al. Tumor doubling time and prognosis in lung cancer patients: evaluation from chest films and clinical follow-up study. Jpn J Clin Oncol 1994;24:199-204.

8. Yankelevitz DF, Kostis WJ, Henschke Cl, et al. Overdiagnosis in chest radiographic screening for lung carcinoma: frequency. Cancer 2003;97:1271-5.

9. Hasegawa M, Sone S, Takashima S, et al. Growth rate of small lung cancers detected on mass CT screening. Br J Radiol 2000;73:1252-9.

10. Lindell RM, Hartman TE, Swensen SJ, et al. Fiveyear lung cancer screening experience: ct appearance, growth rate, location, and histologic features of 61 lung cancers. Radiology 2007;242:555-62.

11. Brenner DJ, Hall EJ. Computed tomography: an increasing source of radiation exposure. N Engl J Med 2007;357:2277-84. 